\title{
Design Research on Algorithm of Processing Time, Data Aggregation Time, Packet Delivery Ratio and Energy Consumption
}

\author{
S. Prabhavathi, Kiran S, Channaveerana Gouda
}

\begin{abstract}
This paper presents the preliminary information of research that deals with communication protocol for Wireless Sensor Network (WSN) with retention of energy efficiency. The motive of this research is to accomplish a common goal of energy efficiency, using different forms of methodologies. Hence, the adoption of different methodologies and a common goal of energy efficiency in WSN are achieved. This paper explains the outcome accomplishment the proposed ENLPL Algorithm, Globular topology, load balancing technique of Processing Time, Data Aggregation Time, Packet Delivery Ratio and Energy Consumption pertaining to optimizing energy using probabilistic technique, and Dynamic Reconfiguration. . The work also explains about the comparative analysis among the models to showcase the best scenario of usage in sensorbased applications.
\end{abstract}

Keywords-WSN, Load Balancing, LEACH, Globular Topology

\section{INTRODUCTION}

There are various available energy efficient techniques which are designed for mitigating energy issues associated with WSN nodes. Some of existing approaches are utilized for transmission power control for both wireless networks and WSNs. The goal of existing approaches is to carry out dynamic adjustment of transmission power from the sensor node in order to maintain a good communication quality between a transceiver pairs associated with two nodes and concept also includes power optimization with respect to throughput enhancement and efficient data delivery ratio. Typically, a sensor radio has three working modes they are transmission, gathering and static listening. The general studies towards energy efficiency shows that a sensor depletes energy both in active and passive states. In active state, a node will spend energy for forwarding the data as well as in various other energy parameters such as receiving, amplification and energy spent due to circuitry operation in a node etc. In a passive state, a sensor node can switch off its radio in order to save power, but it does not do so completely. In fact, in order to wake up a node in sleep mode, a discrete format of a control message is required which also require additional energy.

Power could be either rest / wakeup principle operation on the highest point of a Multiple Access Control (MAC) principle. A few standards can be additionally used to

Revised Manuscript Received on July 10, 2019.

Dr. S. Prabhavathi, Professor Dept of ECE, RYMEC Ballari. Karnataka India.

Kiran S, UG Scholar, SJBIT Bengaluru, Karnataka India.

Channaveerana Gouda, Asst. Professor Dept of ECE, RYMEC

Ballari, Karnataka India. choose which sensor to initiate / deactivate and when. In such manner, topologies conventions can be extensively ordered in the accompanying two classifications, they are area driven conventions characterize which node to turn on and when. For example, span is an availability driven convention that adaptively chooses facilitators of all sensors in the system and Adaptive Self-Configuring Sensor Networks Topologies (ASCENT), area driven topology control conventions particularly oblige that sensor nodes regarding their position. This is for the most part accomplished by furnishing sensors with a GPS unit.

The sensor should wake up just when another sensor needs to speak with it. The principle issue in this regard is to ensure an inter node communication system for a proper routing as well as scheduling for incoming traffic states. Such schemes ordinarily utilize numerous radios with distinctive battery / execution tradeoffs that are low rate and low power radio for flagging and a high rate for information correspondence. Regularly, sensor wake up as indicated by a wakeup plan and stay dynamic for a brief while interim to speak with their neighbors. At that point, they go to rest until the following meeting time. At last, an offbeat rest / wakeup convention may be utilized. With such conventions, a sensor can wake up when it needs and still has the capacity to perform communication with its neighbors. A technique called as Scanty Topology and Energy Management (STEM) utilizes two distinct radios for wakeup sign and information parcel transmissions, separately. Therefore, such conventional scheme offer more symptomatic solution rather than corrected solution for supporting energy efficient routing along with load balancing.

Usage of MAC based conventions for developing a routing protocol for conserving the constantly depleting energy is widely seen in sensor network. However, there is also a contradiction in MAC conventions which are Carrier Sense Multiple Access (CSMA) or Carrier Sense Multiple Access/Collision Avoidance (CSMA/CA), oblige no coordination among sensors getting to the channel. The center point at which a sensor needs to send information will compete for the remote channel. Impacting sensors will back off for an irregular length of time before endeavoring to get the channel once more. The next section discusses some of the standard techniques used in routing protocols. 


\section{DESIGN RESEARCH ON ALGORITHM OF PROCESSING TIME, DATA AGGREGATION TIME, PACKET DELIVERY RATIO AND ENERGY CONSUMPTION}

\section{LITERATURE SURVEY}

Prolonging the life time of a sensor network has become more challenging now a day's [1]. Existing research trends highlight that sensor nodes placed in a particular deployment area form a cluster.The cluster head in a cluster which consists of more powerful and computationally efficient resources [2]. The entire cluster heads gather the collected information and retransmit it to a sink node which is also an electronic device used for processing of huge amount of collected data and sending it to the monitor station via a gateway node [3]. Wireless sensor nodes could be utilized for more than one tasks basically it performs multitasking as an example sensor nodes sense and gathers various environmental attributes, perform data acquisition, aggregation with respect to various filtering conditions.

Encryption and decryption also performed before transmitting the data towards a sink/gateway node or some other corresponding nodes of a particular sensor network [4]. Various load balancing techniques have been designed for creating an energy efficient route in between source and destination node in order to enhance the network lifetime [5]. Balamurugan [6] presented a routing protocol called as Fitness-based Routing Protocol (FRP) to improve battery usage during data transmission. The battery utilization of sensors is diminished by selecting the sensors with least jump tally and separation. Genetic calculation is utilized as a streamlining procedure to locate the fitted sensors in view of its energy conservation. The FRP could expand the system lifetime and throughput contrasting with alternate conventions.

Fu et al. [7] proposed another enhanced calculation of LEACH convention which is expected to adjust the battery utilization of the whole system and expand systems life. The new calculation is imitated by MATLAB recreation stage, the outcome results show that both battery effectiveness and systems lifetime are superior to anything that of LEACH Protocol. Kumar et al. [7] presented an innovative Energy Efficient Clustering and Data Aggregation (EECDA) conventional system for the heterogeneous WSNs which joins the idea of energy efficient cluster based transmission and information collection to accomplish a superior execution regarding lifetime and reliability. EECDA convention incorporates a latest cluster head.

Ghotekar and Mehetre [8] reviewed systems which are utilized as a part of remote sensor system for load balancing. Remote sensor system has diverse nodes with distinctive sort of vitality which can enhance the systems lifetime.

\section{RESEARCH METHODOLOGY}

ENLPL has adopted the research methodology of analytical and empirical approach. The core agenda was to ensure maximum and effective participation of sensor nodes in selection process of cluster leader using probabilistic logic. Accomplishment of this agenda in return gives an energy efficient communication protocol and its optimization in WSN. The schematic diagram of the proposed ENLPL scheme is highlighted in Figure 1. The initial stage of methodology consists of designing the network model, where aggregated data from sensors are forwarded to the base station via cluster leader. A hierarchical clustering technique is applied owing to its energy efficiency characteristics with respect to remnant power of sensor nodes.

In order to increase the level of difficulty in order to map with real-time situation, the proposed system will consider initializing with lesser value of initial energy and will be allocated with only $5 \%$ probability of selection of cluster leader for every increasing round. The most critical perspective of the design principle is to develop a probabilistic reasoning scheme that can effectively choose the optimal cluster leader and controls other nodes to become cluster leader in consecutive rounds. Candidate nodes have fair amount of energy in order to make them self eligible to become cluster leader in the next round.

This System also considers non-candidate node as those nodes which have significantly less amount of energy and have less possibility of becoming a cluster leader. However, there are fair chances of non-candidate nodes to become candidate nodes in the next round in progression. Prime factor of selection process is again the higher residual energy.

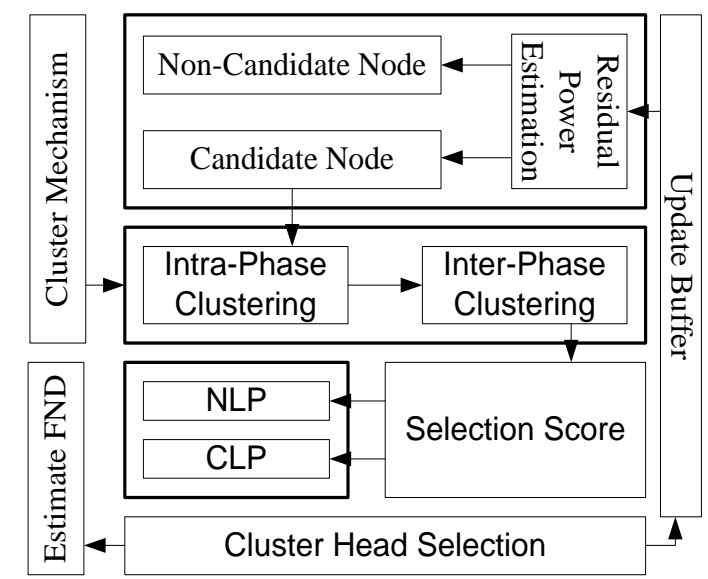

Figure 1: Schema of Proposed ENLPL System

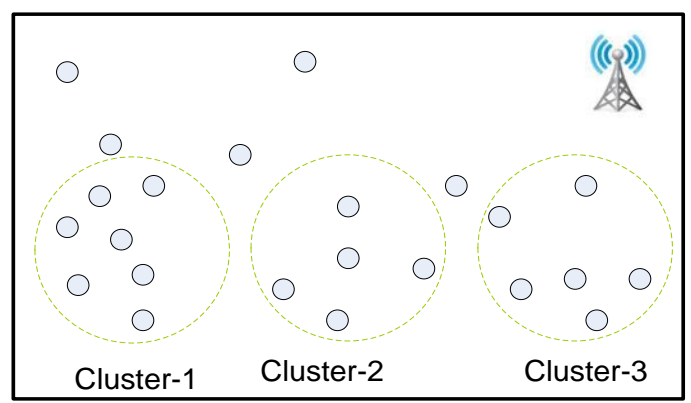

Figure 2: Formations of Clusters

Figure 3 shows the comparative performance analysis of the proposed ENLPL with the LEACH protocol. For LEACH algorithm, outcome shows that with the increasing number of iteration, number of alive nodes are found to be uniform till $100^{\text {th }}$ iteration and then it starts a stiff fall of peaks representing higher extent of energy depletion. Under similar condition, proposed ENLPL has shown the linearlity behaviour of energy curve till $550^{\text {th }}$ iterations and started degrading after that. 


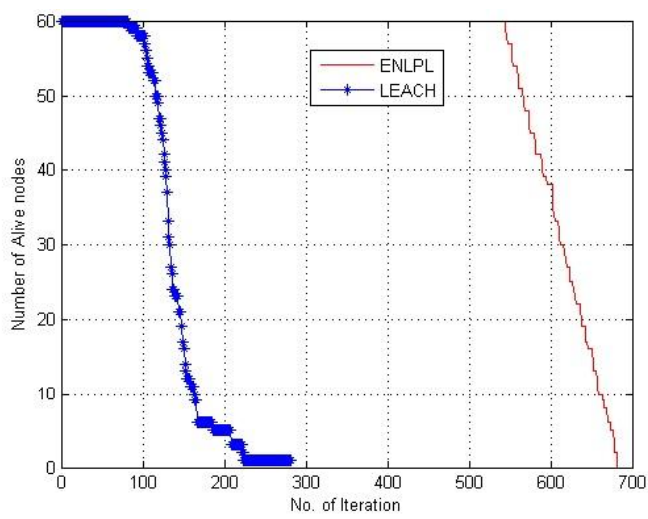

Figure 3: Number of Alive Nodes vs. Iterations

The outcome shows that LEACH has stiff declination of residual energy till $140^{\text {th }}$ iteration that it maintains uniformity till $290^{\text {th }}$ iteration where it witness $100 \%$ of node death. ENLPL has also exhibited degradation of residual energy, but $100 \%$ of the node death was only witnessed in near to $700^{\text {th }}$ iteration as shown in figure 4.

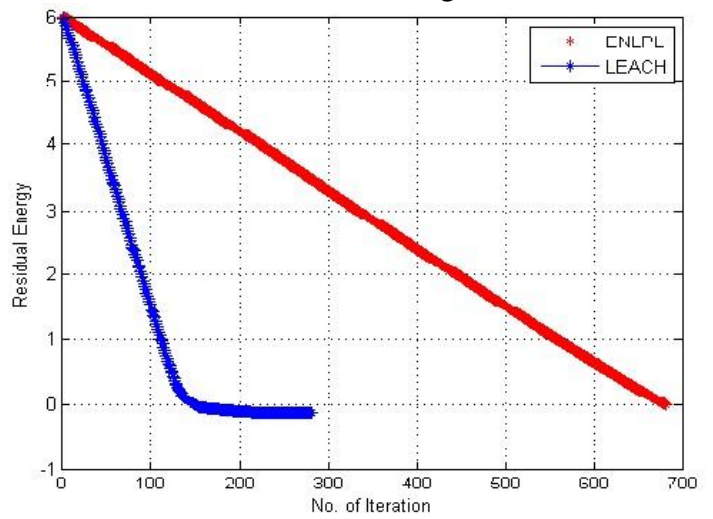

Figure 4: Impact of Residual Energy on Iterations

Table 1 show that occurrences of FND surfaces for proposed ENLPL system for approximately on 656 iterations, whereas for LEACH, it was found on 378 iterations. The outcome will mean that proposed ENLPL can offer $65.6 \%$ of energy conservation whereas LEACH can offer $37.8 \%$ of the energy conservation performance under similar simulation environment.

Table 1: Comparison of FND parameter for ENLPL and LEACH protocol

\begin{tabular}{|c|c|}
\hline ENLPL & LEACH \\
\hline 610 & 340 \\
\hline 625 & 342 \\
\hline 635 & 359 \\
\hline 645 & 365 \\
\hline 667 & 370 \\
\hline 668 & 377 \\
\hline 670 & 389 \\
\hline 675 & 395 \\
\hline 679 & 421 \\
\hline 689 & 425 \\
\hline 656 & 378 \\
\hline ES $_{\mathrm{ENLPL}}=[656 / 1000] * 100$ & $\mathrm{ES}_{\mathrm{LEACH}}=[378 / 1000] * 100$ \\
$=65.6 \%$ & $=37.8 \%$ \\
\hline
\end{tabular}

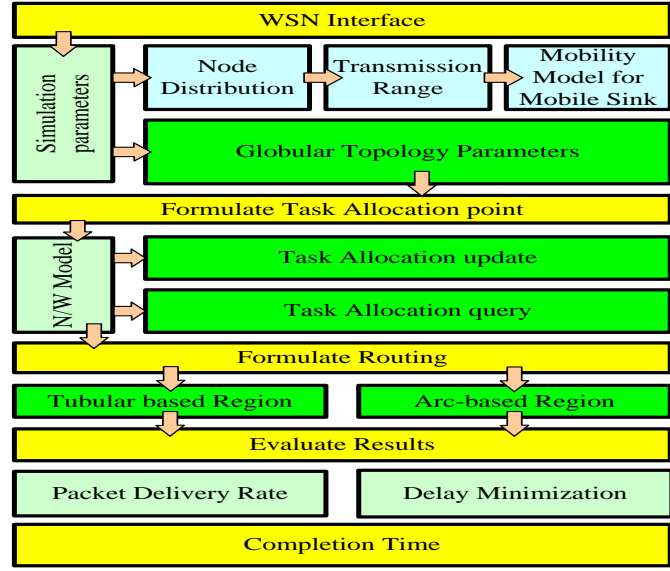

Figure 5: Proposed System Schema

The outcome is also evaluated for propagation delay. The core reason behind the degraded outcome of Petrioli et. al. is because of non-inclusion of the mobile base station and more iterative operations with the base station using greedy approach resulting in more computational time that degrades packet delivery ratio of the existing system as shown in Figure 6.

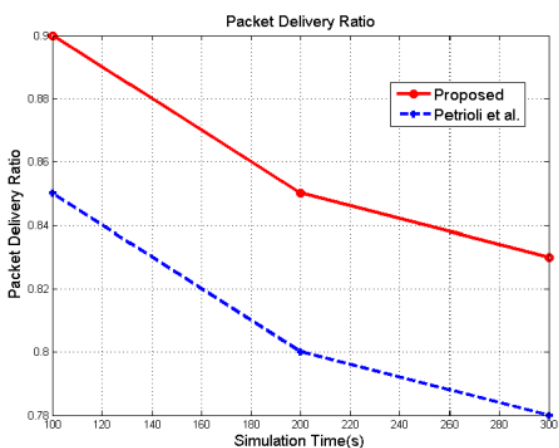

Figure 6: Packet Delivery Ratio

The performance of the delay estimation is carried out as graphically presented in Figure 7. Curve shows the level of delay that can be minimized using both techniques in comparative work. System evaluates the cumulative difference of time consumed for carrying out the data aggregation method in the universal radio model that is considered in static sensors with one base station. Owing to more inclusion of computational time in the Petrioli et al [9], delay estimates are quite high leading to enough computational burdens at the time of communication

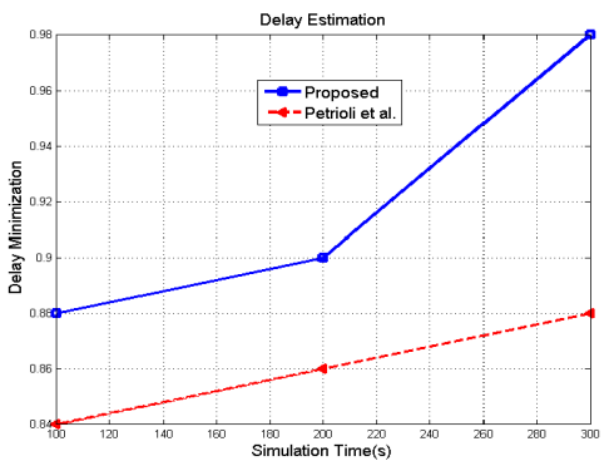

Figure 7: Propagation Delay Analyses 


\section{DESIGN RESEARCH ON ALGORITHM OF PROCESSING TIME, DATA AGGREGATION TIME, PACKET DELIVERY RATIO AND ENERGY CONSUMPTION}

The outcome of the work is evaluated using the job completion time as shown in Figure 8. Outcome shows that proposed system consumes lesser processing time to perform data aggregation. Prior outcomes already infer the better communication outcomes for the proposed work as compared to the standard work undertaken by the Petrioli et al. [9] approach

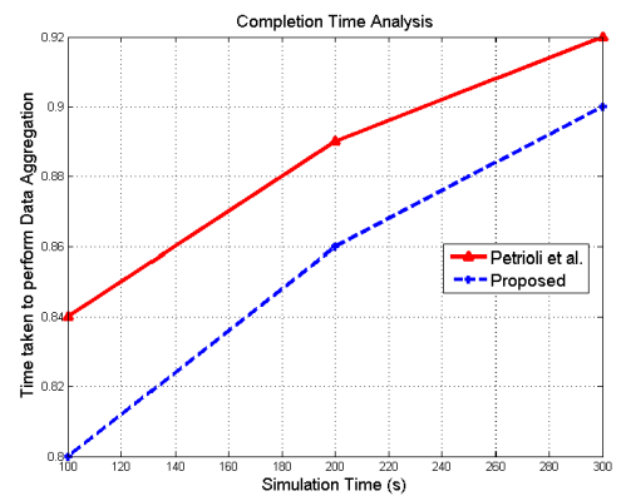

Figure 8: Analysis of Job Completion Time

\begin{tabular}{|c|c|c|c|}
\hline \multicolumn{4}{|c|}{$\begin{array}{c}\text { Initialize Simulation Parameters } \\
\text { (Nodes, Clusters, Time, Simulation Area) }\end{array}$} \\
\hline \multicolumn{4}{|c|}{ Create Network Model } \\
\hline $\begin{array}{l}\text { Member } \\
\text { Node }\end{array}$ & $\begin{array}{l}\text { Aggregator } \\
\text { Node }\end{array}$ & $\begin{array}{r}\text { Rout } \\
\text { Age }\end{array}$ & $\begin{array}{l}\text { Base } \\
\text { Station } \\
\end{array}$ \\
\hline \multicolumn{4}{|c|}{$\begin{array}{c}\text { Create Energy Model } \\
\left(\mathrm{E}_{\mathrm{TX}}, \mathrm{E}_{\mathrm{RX}}\right) \\
\end{array}$} \\
\hline $\begin{array}{c}\text { Data Packet } \\
\text { Size }\end{array}$ & Data rate & $\begin{array}{l}\text { Sup } \\
\text { Vol } \\
\end{array}$ & Overhead \\
\hline \multicolumn{2}{|c|}{$\begin{array}{l}\text { Current Drawn from } \\
\text { Communication Module }\end{array}$} & \multicolumn{2}{|c|}{$\begin{array}{l}\text { Current Drawn from } \\
\text { Sensor Module }\end{array}$} \\
\hline \multicolumn{3}{|c|}{$\begin{array}{c}\text { Decide number of clusters for } \\
\text { proposed system }\end{array}$} & $2^{c-1}-1$ \\
\hline \multicolumn{3}{|c|}{$\begin{array}{c}\text { Calculate maximum number of } \\
\text { nodes in each clusters }\end{array}$} & $\eta_{i}=2^{i-1}$ \\
\hline \multicolumn{4}{|c|}{ Perform Data Aggregation } \\
\hline \multicolumn{4}{|c|}{ Calculate Total Energy for all Clusters } \\
\hline \multirow{2}{*}{$\begin{array}{c}\text { Compare with } \\
\text { LEACH }\end{array}$} & \multicolumn{3}{|c|}{ Cumulative Energy Consumption } \\
\hline & \multicolumn{3}{|c|}{$\begin{array}{c}\text { Cumulative Data Aggregation } \\
\text { Duration }\end{array}$} \\
\hline
\end{tabular}

Figure 9: Schema of the Proposed Work

Table 2 shows the outcome for processing time of only the algorithms ignoring the statement for generating graphical outcomes in MATLAB. The evaluation is carried out from 30 to 1000 iterations to check if the increasing iterations have significant impact on processing time. It is quite understood concept that increase of iteration will increase traffic load resulting in increase in processing time. However, the emphasis is only given to understand which technique is better.

Table 2: Comparative Analysis of Execution Time

\begin{tabular}{|c|c|c|c|c|c|c|}
\hline \multirow{3}{*}{ Iterations } & \multicolumn{5}{|c|}{ EXECUTION TIME } \\
\cline { 2 - 7 } & \multicolumn{3}{|c|}{ LEACH } & \multicolumn{3}{c|}{ Proposed System } \\
\cline { 2 - 7 } & Core-i3 & Core i5 & Core-i7 & Core-i3 & Core i5 & Core-i7 \\
\hline 30 & 0.727 & 0.633 & 0.411 & 0.047 & 0.021 & 0.012 \\
\hline 100 & 0.754 & 0.657 & 0.419 & 0.063 & 0.024 & 0.012 \\
\hline 170 & 0.734 & 0.659 & 0.435 & 0.080 & 0.036 & 0.014 \\
\hline 240 & 0.756 & 0.688 & 0.477 & 0.092 & 0.048 & 0.019 \\
\hline 310 & 0.771 & 0.692 & 0.481 & 0.109 & 0.056 & 0.023 \\
\hline 380 & 0.800 & 0.751 & 0.521 & 0.125 & 0.064 & 0.028 \\
\hline 450 & 0.809 & 0.755 & 0.521 & 0.146 & 0.069 & 0.035 \\
\hline
\end{tabular}

\begin{tabular}{|c|c|c|c|c|c|c|}
\hline 520 & 0.831 & 0.768 & 0.535 & 0.157 & 0.072 & 0.039 \\
\hline 590 & 0.8705 & 0.789 & 0.552 & 0.174 & 0.072 & 0.044 \\
\hline 660 & 0.872 & 0.792 & 0.569 & 0.187 & 0.077 & 0.048 \\
\hline 730 & 0.936 & 0.802 & 0.578 & 0.209 & 0.081 & 0.055 \\
\hline 1000 & 0.994 & 0.834 & 0.588 & 0.262 & 0.093 & 0.061 \\
\hline
\end{tabular}

Table 3: Impact of Processor on Execution Time

\begin{tabular}{|c|c|c|}
\hline \multirow{2}{*}{ Iterations } & \multicolumn{2}{|c|}{ EXECUTION TIME } \\
\cline { 2 - 3 } & LEACH & Proposed System \\
\hline 30 & 0.727 & 0.047 \\
\hline 100 & 0.754 & 0.063 \\
\hline 170 & 0.734 & 0.080 \\
\hline 240 & 0.756 & 0.092 \\
\hline 310 & 0.771 & 0.109 \\
\hline 380 & 0.800 & 0.125 \\
\hline 450 & 0.809 & 0.146 \\
\hline 520 & 0.831 & 0.157 \\
\hline 590 & 0.8705 & 0.174 \\
\hline 660 & 0.872 & 0.187 \\
\hline 730 & 0.936 & 0.209 \\
\hline 1000 & 0.994 & 0.262 \\
\hline
\end{tabular}

Table 3 highlights the impact of the different types of frequently used processors although, it is known that there will be increase of computational capability using improved versions of processor, but it is important to understand the difference. The numerical outcome shows that with the increase of processing capabilities, proposed processing time reduces drastically around $10-20 \%$ approximately.

\section{RESULT DISCUSSION}

The outcome presented in Figure 9 shows that ENLPL is highly appropriate for sensors which require processing of critical information. Various applications in WSN pertaining to habitat and environmental monitoring require sensors to process the data faster owing to the massiveness of the data. So that processed data could be easily compatible with load balancing algorithms in reality. Hence, the model of ENLPL is highly recommended for usage in such application scenario.

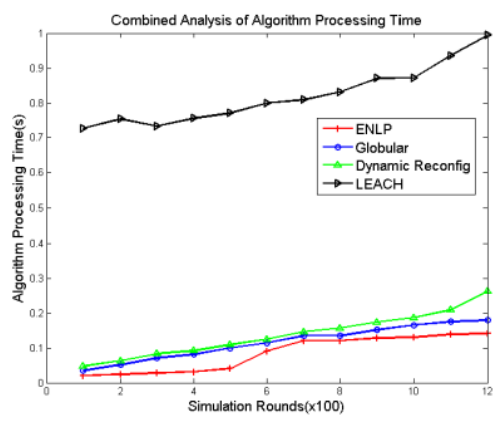

Figure 10: Joint Outcome of Algorithm Processing Time 
The outcome exhibited in Figure 10 clearly shows that Globular topology exhibits superior performance in comparison to dynamic reconfiguration and ENLPL. The prime reason behind this is globular topology introduces a completely novel method to search the destination node using dual-steps of search mechanism. Although, it consumes little processing time in initial search levels, but the more the process runs, the topology posses more heuristic data of routing. Dynamic routing and ENLPL have better time-based performance but owing to much optimization and agent usage, the packet delivery ratio is not as better as globular.

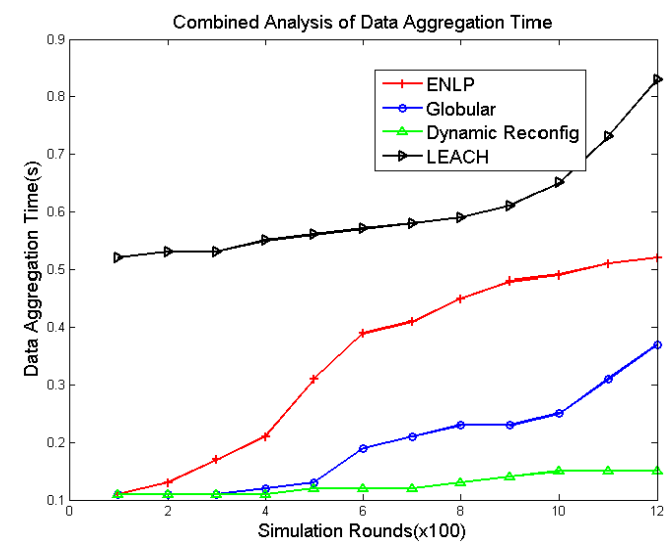

Figure 11: Joint Outcome of Data Aggregation Time

Assessing data aggregation time gives a better idea of sensor network efficiency with respect to realistic scenario mean capability of the sensor to transmit the data to sink faster. The outcome shown in Figure 11 highlights that curve corresponding to dynamic reconfiguration has highly minimized data aggregation time and is better in contrast to data aggregation time for ENLPL and globular topology. ENLPL could have better algorithm processing time but it performs optimization in multiple level which calls for increasing time to actually transmit the data from one aggregator node to sink. Owing to simpler search technique and mobile sinks, globular topology could deliver the data faster compared to ENLPL. However, Globular topology uses search-based optimization in two levels and doesn't support dynamic reconfiguration. This results in globular topology not to excel better data aggregation time in contrast to dynamic reconfiguration.

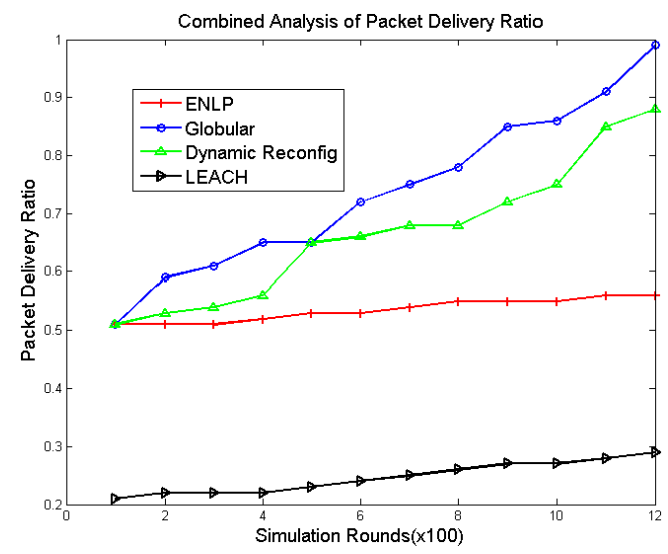

Figure 12: Joint Outcome of Packet Delivery Ratio of application. Reduced data aggregation time will only

Packet delivery ratio is the most appropriate performance parameter to understand the effect of load balancing technique developed in proposed work. The outcome exhibited in Figure 12 clearly shows that Globular topology exhibits superior performance in comparison to dynamic reconfiguration and ENLPL. The prime reason behind this is globular topology introduces a completely novel method to search the destination node using dual-steps of search mechanism. Although, it consumes little processing time in initial search levels, but the more the process runs, the topology posses more heuristic data of routing. Dynamic routing and ENLPL have better time-based performance but owing to much optimization and agent usage, the packet delivery ratio is not as better as globular.

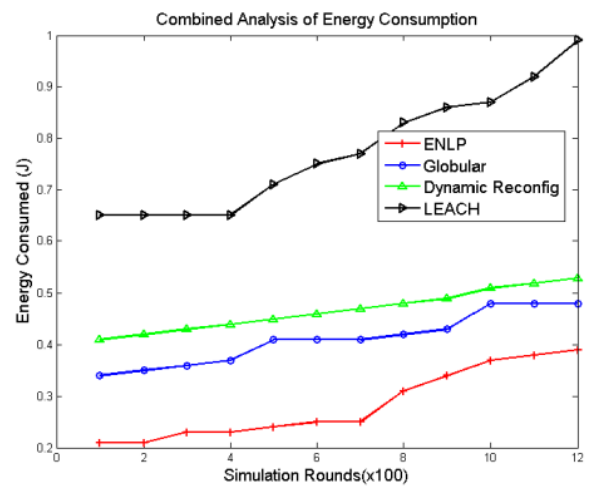

Figure 13: Joint Outcome of Energy Consumption

\section{CONCLUSION}

This paper presents the combined outcomes of the proposed system with special emphasis on three significant models that is optimizing energy using probabilistic technique, globular topology and dynamic reconfiguration. The outcome shows the comparative performance analysis of the proposed system with conventional LEACH protocol. The paper also explains about the comparative analysis among the models to showcase the best scenario of usage in sensor-based applications.

\section{REFERENCES}

1. Kumar D., Patel R. and Aseri T.C: Prolonging Network Lifetime and Data Accumulation in Heterogeneous Sensor Networks, Int. Arab J. Inf. Technol, 7(3), (2010), 302309.

2. Kumar A., Kumar V. and Chand N.: Energy Efficient Clustering and Cluster Head Rotation Scheme for WSNs, (IJACSA) International Journal of Advanced Computer Science and Applications, 3 (5), (2011).

3. Taruna S., Kohli S. and Purohit G.N : Distance Based Energy Efficient Selection Of Nodes To Cluster Head In Homogeneous WSNs, International Journal of Wireless \& Mobile Networks, 4(4), 243, (2012).

4. Sasikala K. and Geetha M.: A Review on Energy Efficient Secure Routing For Data Aggregation In WSNs, International Journal of Engineering Trends and Technology (IJETT), 4 (Retrieved, 2nd Nov, 2015).

5. Bouabdallah F., Bouabdallah N. and Boutaba R.: LoadBalanced Routing Scheme for Energy-Ffficient WSNs, In Global Telecommunications Conference, IEEE GLOBECOM, (2008), 1-6. 
6. Balamurugan A.: An Energy Efficient Fitness based Routing Protocol in WSNs, ICTACT Journal on Communication Technology, 5(1), (2014).

7. Fu, C., Jiang Z., Wei W.E.I. and Ang WEI. An Energy Balanced Algorithm of LEACH Protocol in WSN, IJCSI International Journal of Computer Science Issues 10(1), (2013), 354-359.

8. Ghotekar R.K. and Mehetre D.C.: Load Balancing for Achieving the Network Lifetime in WSN-A Survey, (2014).

9. Petrioli C., Nati M., Casari P.: ALBA-R: Lead-Balancing Geographic Routing Around Connectivity Holes in WSN, IEEE Transactions on Parallel and Distributed System,(2014),529-53 